\title{
L'ENEMIGO DE NATURA HUMANA NELLA PROSPETTIVA DI IGNAZIO DI LOYOLA
}

The Enemy of Human Nature from Ignatius of Loyola's Perspective

Tiziano Ferraroni *

SOMMARIO: A differenza di molti autori spirituali della sua epoca o delle epoche precedenti, Ignazio di Loyola predilige, per parlare del demonio, il termine "nemico" o ancora di più l'espressione "nemico della natura umana". In questo nostro contributo investighiamo tale espressione: ne analizziamo le ricorrenze negli scritti ignaziani ed esploriamo le fonti da cui Ignazio potrebbe averla ereditata. La difficoltà a rintracciarne l'origine ci spingerà ad approfondire separatamente il significato di "nemico" e di "natura umana" nell'orizzonte semantico/culturale di Ignazio, per poi congiungere questi termini e formulare qualche ipotesi sull'accezione che Ignazio attribuiva all'espressione indagata. Ne emerge la visione di un combattimento escatologico che si svolge nel cuore di ogni uomo e di ogni donna, e in cui ciò che è in gioco è proprio l'interpretazione della natura umana e la piena realizzazione dell'uomo in Dio.

PAROLE CHIAVE: Nemico della natura umana. Demonio. Ignazio di Loyola. Combattimento escatologico. Modernità.

ABSTRACT: Unlike many spiritual authors of his time or earlier times, Ignatius of Loyola prefers to use the term "enemy" or, even, the expression "enemy of human nature", when speaking of the devil. We investigate such expressions in this paper: we analyse their occurrence in Ignatian writings and explore the sources from which Ignatius may have drawn. The difficulty in tracing their origins leads us to investigate separately the meaning of "enemy" and "human nature" in Ignatius' semantic/cultural horizon, and then to combine these terms and formulate some

* Pontificia Facoltà Teologica dell'Italia Meridionale, sezione san Luigi, Napoli, Italia. 
hypotheses about the meaning Ignatius gave to the expressions under investigation. What emerges is a vision of an eschatological combat taking place in the heart of every man and woman, in which what is at stake is precisely the interpretation of human nature and the full accomplishment of man in God.

KEYWORDS: Enemy of Human Nature. Demon. Ignatius of Loyola. Eschatological Combat. Modernity.

\section{Introduzione}

L

a tradizione cristiana si è sempre preoccupata di mettere in guardia l'uomo da una presenza maligna che, pur non potendo competere con Dio in dignità e potenza, si impiega a guastare la sua opera, cercando di ostacolare la salvezza dell'uomo e più in generale il compimento della creazione. Tante sfaccettature e tonalità del modo di agire di questa presenza sono emerse lungo i secoli, e tanti sono i nomi che le sono stati attribuiti. La maggior parte di essi sono nomi comuni, più o meno figurativi, talvolta personificati, che esprimono ognuno qualcosa del suo modo di agire: Satana (l'accusatore), diavolo (il divisore), il maligno, il serpente, il dragone, il demonio, ecc. (LYONNET, 1957, v. 3, p. 142-145). C'è poi l'appellativo "Lucifero", che significa "portatore di luce", che nel corso dei secoli è diventato il nome proprio dell'angelo ribellatosi a Dio per orgoglio ${ }^{1}$. Gli sono così riconosciute una volontà e una libertà proprie, e quindi la dignità di creatura intelligente, di persona. Aggiungiamo che a volte se ne parla al singolare, altre volte al plurale (demoni, diavoli, Principati e Potestà, ecc.). Sembra che tanti nomi e tante rappresentazioni siano necessarie per designare una presenza che non ha nome e non ha volto, la cui tattica consiste proprio nello sgusciare da ogni tentativo di comprensione, e di presa.

Ignazio di Loyola, uomo ancorato al sentire nella Chiesa (ES, n. 352) $)^{2}$, per di più, imbevuto della sensibilità medievale che dava grande rilievo alla lotta contro il demonio, accoglie senza riserve la dottrina e le rappresentazioni trasmessegli: lo mostra il fatto che, quando nella Prima Settimana degli Esercizi Spirituali l'esercitante è invitato a meditare sulla dinamica del peccato a partire dalla caduta degli angeli, le poche parole che il libretto consacra a questo episodio rispecchiano la demonologia classica

\footnotetext{
${ }^{1}$ Il sostantivo "lucifero" è attribuito già nel libro di Isaia ad un essere caduto dal cielo (Is 14,12), mentre nella seconda lettera di Pietro, lucifero - lucifer nel latino della Vulgata, $\varphi \omega \sigma \varphi$ óoos nell'originale greco - sta ad indicare il Cristo atteso $(2 \mathrm{Pt} 1,19)$. Nei primi secoli cristiani "lucifero" era quindi un titolo di Cristo, al punto da essere un nome di battesimo. ${ }^{2}$ Per quanto riguarda i testi di Ignazio, ci riferiamo agli originali in castigliano, riportati in BAC (IPARRAGUIRRE; DE DALMASES; JURADO, 1991). La traduzione in italiano è nostra.
} 
(ES, n. 50). Se è vero che Ignazio assume la concezione demonologica tradizionale, non tutti i termini hanno però lo stesso peso per lui: non è un caso che la parola "Satanás" appaia raramente nei suoi scritti ${ }^{3}$, che il termine "diablo" non esca mai dalla sua penna ${ }^{4}$, e che invece compaia a più riprese il vocabolo "demonio"s. C’è poi l'espressione "mal espíritu"6, che per Ignazio corrisponde allo spirito del demonio, che insinua nel cuore dell'uomo pensieri nocivi, contrapposti a quelli buoni suggeriti dallo spirito di Dio (R, n. 8).

Ciò che attira la nostra attenzione, però, è il fatto che il termine che Ignazio predilige per descrivere questa presenza maligna è quello di "enemigo" Talvolta a questo termine egli aggiunge la specificazione "de natura humana", con alcune variazioni ${ }^{8}$. Una volta sola appare il sinonimo "adversario" (ES, n. 13). Osserviamo quindi che, per quanto Ignazio si ponga in continuità con la tradizione, il fatto che privilegi certi termini rispetto ad altri è rivelatore di una sensibilità se non addirittura di un'opzione determinata. Parlare del demonio nei termini di "nemico" significa assumere una prospettiva particolare, e parlarne nei termini più specifici di "nemico della natura umana" lascia intravvedere una certa visione dell'uomo, di Dio e del demonio, e una certa comprensione del loro modo di agire e di interagire. Se poi consideriamo che l'espressione "enemigo de natura humana" non si incontra facilmente negli scritti di quell'epoca, non pochi interrogativi si schiudono: cosa intende Ignazio quando parla del "nemico della natura umana"? Le ricorrenze di questa espressione, considerate nel loro contesto, cosa ci rivelano della concezione antropologica e teologica di Ignazio? Quali implicazioni nella comprensione della battaglia che ogni

\footnotetext{
${ }^{3}$ La Concordancia ignaciana (ECHARTE, 1996) non riporta alcuna ricorrenza di questa parola. Essa appare una volta negli Avisos de N. Bto Pe Ignacio (MI, reglas, 141-143, [9]), citando san Paolo: "Satana che si trasforma in angelo di luce" (2 Cor 11,14). Anche in una lettera al fratello Martín (Epp. 1, p. 79-83) compare una citazione di san Paolo in cui si parla di un "emissario di Satana" (2 Cor 12,7).

${ }^{4}$ Ancora una volta facciamo riferimento alla Concordancia ignaciana e alla BAC.

${ }^{5} 2$ volte nel Racconto del Pellegrino, 3 volte nelle Costituzioni, almeno 17 volte nelle lettere riportate dalla BAC. Negli Esercizi la parola appare 2 volte, sempre al plurale.

${ }^{6}$ Letteralmente quest'espressione compare solamente nel Diario (2 volte) e nelle Lettere: per esempio ne troviamo 3 ricorrenze nella lettera indirizzata a Francisco de Borja del luglio 1549 (Epp. 12, p. 632-654). Negli Esercizi i riferimenti allo spirito cattivo sono numerosi, anche se a volte appare solo il pronome "malo" in opposizione allo spirito buono, come per esempio: "nella consolazione di guida e ci consiglia di più il buono spirito, mentre nella desolazione il cattivo" (ES, n. 318). Così in (ES, n. 32, 336). Nei numeri $(331,332,335)$ l'aggettivo o pronome "malo" è riferito all'angelo, in contrasto al "buen ángel". Cf. (PALACIO, 2007, v. 2, p. 1175-1180).

${ }^{7}$ Il termine compare 37 volte negli Esercizi. Di queste, però, 6 volte non sono riferite alla presenza demoniaca, ma ad altri nemici. Nelle Costituzioni la parola "nemico" riferita al demonio appare 3 volte. Troviamo un riferimento anche nel Racconto del Pellegrino e uno nel Diario spirituale (Cf. ECHARTE, 1996, p. 447-448).

${ }^{8}$ Non ci soffermiamo ora a specificare in numero delle ricorrenze di questa espressione e delle sue variazioni, perché il suo studio sarà l'oggetto di questo nostro contributo.
} 
uomo e ogni donna sono chiamati a combattere? E infine, tale espressione è ancora eloquente oggi?

In questo articolo ci concentreremo soprattutto sull'espressione "enemigo de natura humana". Dopo aver presentato le sue ricorrenze negli scritti di Ignazio, indagheremo sulle sue possibili origini. In seguito la scomporremo e cercheremo di cogliere cosa Ignazio intenda rispettivamente per "nemico" e per "natura umana", a partire dalla cultura sociale e teologica di cui era impregnato, oltre che dall'analisi dei suoi stessi scritti. Solo allora ci sarà possibile ricomporre l'espressione e comprenderla nella sua integralità e nella sua portata, per il tempo di Ignazio così come per il nostro tempo.

\section{Ricorrenze e origine dell'espressione "enemigo de natura humana"}

\subsection{Ricorrenze}

Nel libretto degli Esercizi Spirituali, l'espressione "enemigo de natura humana" appare sette volte ${ }^{9}$. A queste ricorrenze bisogna aggiungerne altre due che riportano alcune varianti: al numero (n.136) appare l'aggettivo possessivo "nostro" e si assiste all'inversione tra il sostantivo "natura" e l'aggettivo "humana": "enemigo de nuestra humana natura"; al numero (n. 333) Ignazio modifica il termine della specificazione: "enemigo de nuestro provecho y salud eterna". Nelle Costituzioni troviamo una sola ricorrenza di questa locuzione, con l'aggiunta dell'articolo determinativo "enemigo de la natura humana" (Co, n. 553). Al numero (n. 622) delle Costituzioni il nemico diventa "enemigo de Christo N. S.", con riferimento esplicito alla parabola evangelica della zizzania.

Se consideriamo le Lettere e Istruzioni, constatiamo che Ignazio amava servirsi dell'espressione sulla quale verte la nostra ricerca. E interessante notare che essa appare già nella prima lettera che conserviamo di Ignazio, scritta a Inés Pascual nel 1524, durante la sua permanenza a Barcellona ${ }^{10}$. Un'altra ricorrenza si trova in una lettera del 12 febbraio 1536, indirizzata a Jaime Cassador $^{11}$, e un'altra ancora nella missiva del 18 giugno 1536, inviata a sor Teresa Rejadell ${ }^{12}$. In quest'ultima Ignazio aggiunge l'aggettivo "tutta":

\footnotetext{
${ }^{9}$ ES n. 7, 10, 135, 325, 326, 327, 334.

${ }^{10}$ Barcellona, 6 dicembre 1524 (Epp. 1, p. 71-73).

${ }_{11}$ Venezia, 12 febbraio 1536 (Epp. 1, p. 93-99). En las Monumenta ignaziana hay 12 tomos sobre las Letras y instrucciones. 1 indica el primer tomo y 93-99 son las páginas.

${ }^{12}$ Venezia, 18 giugno 1536 (Epp. 1, p. 99-107).
} 
"enemigo de toda natura humana". L'espressione ritorna in più lettere ${ }^{13}$. Quella del luglio 1549, che ha come destinatario Francisco de Borja ${ }^{14}$, ci fornisce un'informazione in più per la nostra investigazione: il testo originale, redatto da Polanco su commissione di Ignazio, conserva le correzioni autografe di quest'ultimo (BAC, p. 842). In esso è evidente la cancellazione da parte di Ignazio della parola "demonio", per sostituirvi di suo pugno l'espressione "enemigo de natura humana" (BAC, p. 847, nota 22).

Il fatto che Ignazio ricorra più volte a quest'espressione è segno del valore che le attribuisce, e il fatto che sia stato lui stesso a modificare la scrittura di Polanco rimpiazzando "demonio" con "nemico della natura umana" significa che per lui i due termini non erano esattamente equivalenti o che, per lo meno, nel contesto di quella lettera, gli sembrava più pertinente parlare di "nemico della natura umana". Il fatto poi che Ignazio, oltre a servirsi spesso di quest'espressione, si permetta di creare alcune varianti, è segno che se ne era appropriato intimamente. Sorge spontanea, a questo punto, la domanda: "da chi l'aveva ereditata?".

\subsection{Ipotesi sull'origine}

La ricerca effettuata ci ha mostrato che non è per nulla scontato risalire all'origine di questa espressione. Una prima indagine effettuata sul web, in diverse lingue, dà come risultati soltanto alcune citazioni di Ignazio o di autori che a loro volta citano Ignazio, come se fosse stato lui stesso a coniarla. Ci siamo allora immersi nei testi che sappiamo essere stati fonti di ispirazione per Ignazio: quelli che lui stesso nomina ${ }^{15}$ e quelli che, secondo le ricerche compiute, lo avrebbero influenzato ${ }^{16}$. Senza pretendere di dare una risposta definitiva - l'ampiezza della ricerca rende impossibile

\footnotetext{
${ }^{13}$ A p. Petro de Soto, il 20 febbraio 1546, (Epp. 1, p. 363-365); a p. Bartolomeo Ferron, il 2 marzo 1547 (Epp. 1, p. 460-467); a p. Michele Turriano, il 3 maggio 1547 (Epp. 1, p. 488-490); a p. Joanne Boquet, nel maggio del 1547 (Epp. 1, p. 494-495); a Julio Porcaro, il 18 luglio 1548 (Epp. 2, p. 159-160); a Juan de Avila il 24 gennaio 1549 (Epp. 2, p. 316-317); al Pontefice Giulio III nel 1554 (Epp. 6, p. 443-444).

${ }^{14}$ Luglio del 1549 (Epp. 12, p. 632-654).

${ }^{15}$ Il Racconto del Pellegrino narra del ruolo cruciale che la Vita di Cristo e una Vita dei santi hanno avuto nella conversione del giovane Inigo, convalescente a Loyola (R, n. 5). Si tratta della Vita Christi di Ludolfo di Sassonia e della Legenda aurea di Jacopo di Varazze. Un altro scritto caro a Ignazio, che lo ha accompagnato fin dal tempo di Manresa e di cui egli stesso consiglia la lettura negli Esercizi, è l'Imitazione di Cristo (ES, n. 100). Ignazio lo chiamava familiarmente Gersoncito e, a detta dei primi compagni, lo leggeva quotidianamente (GARCIA MATEO, 2007, v. 2, p. 994-1001).

${ }^{16}$ Un libro che ha senz'altro influenzato Ignazio è stato L'Ejercitatorio di Cisneros. Questo testo, e soprattutto il Compendio, circolava a Montserrat ai tempi in cui Ignazio frequentava il monastero (MELLONI RIBAS, 1998, p. 353- 377). Ci sono autori che mostrano le influenze che alcuni padri come Origene o Cassiano avrebbero avuto su Ignazio (LIES, 1998, p. 101121; ARZUBIALDE, 1998, p. 123-186). Cassiano è più volte nominato nelle Lettere e Istruzioni, soprattutto in quelle degli ultimi anni, redatte da Polanco.
} 
l'esaustività -, abbiamo riscontrato che, per quanto il termine "nemico" per designare il demonio sia frequente in molti di questi scritti ispiratori, l'unico testo che riporta un'espressione prossima a quella indagata si trova nella Legenda aurea di Jacopo da Varazze: nelle pagine dedicate alla "Passione del Signore" appare due volte l'espressione "nemico del genere umano" - "humani generis inimicus" (IACOPO DI VARAZZE, 1995, p. 285 e 287).

Possiamo supporre che sia proprio dalla Legenda aurea che Ignazio abbia ereditato quest'espressione, pur sostituendo il termine "genere" con il termine "natura"? È anche possibile che quest'espressione circolasse semplicemente nel periodo in cui è vissuto Ignazio. In mancanza di altri indizi, approfondiamo la prima ipotesi. A suo supporto c'è il fatto che il giovane convalescente di Loyola, durante i lunghi mesi passati nella sua stanza, ha avuto a disposizione esclusivamente la Vita di Cristo e la Vita dei santi e che, come ci riferisce l'autore del Racconto del Pellegrino, li ha letti, riletti e ne ha trascritto le parti essenziali in un quaderno ( $R$, n. 11). Non sarebbe strano che proprio durante quei mesi questa espressione sia entrata nel suo orizzonte semantico/linguistico, e che egli se ne sia intimamente appropriato. Un'ulteriore conferma potrebbe venire dal fatto che Ignazio utilizzi questa espressione già nel 1524, cioè negli anni di Barcellona, quando aveva appena cominciato a studiare: il numero di scritti di autori spirituali approcciati fino a quel momento doveva essere senz'altro limitato.

Supposto quindi che la fonte di ispirazione dell'espressione "enemigo de natura humana" sia stata la Legenda aurea, rimane da spiegare come mai Ignazio abbia sostituito il termine "genere" con il termine "natura". Senz'altro non perché la la parola castigliana "genero" fosse estranea al vocabolario di Ignazio: essa compare tre volte negli Esercizi, sempre associata all'aggettivo "humano", là dove si parla della corruzione del genere umano (ES, n. 51) e della salvezza del genere umano (ES, n. 102), e in particolare nella dichiarazione che Ignazio attribuisce alle Persone della Trinità: "Facciamo la redenzione del genere umano" (ES, n. 107). Come ci mostrano questi esempi, quando Ignazio parla di "genere umano" si riferisce al complesso degli uomini e delle donne, all'umanità intera. L'espressione "natura umana" sembra invece indicare per lui una dimensione più essenziale, più intrinseca all'uomo ${ }^{17}$. Notiamo anche che Ignazio avrebbe potuto adottare il termine "naturaleza", che in castigliano era ed è più usuale, piuttosto che il termine "natura", che invece ha una connotazione

\footnotetext{
${ }^{17}$ In una nota relativa all'espressione "enemigo de natura humana", José García de Castro afferma che questo genitivo non è da intendere come "proprietà o relazione (non è nemico degli uomini)", ma come "materia o naturalità", nel senso che il nemico "è composto o prende la forma della natura umana" (GARCIA DE CASTRO, 2001, p. 48, nota 7). Condividiamo la sottolineatura che questa espressione indica qualcosa di più radicale del semplice "prendersela con gli uomini".
} 
latinizzante ${ }^{18}$. Rimane il fatto che, per quanto nella Legenda aurea compaiano spesso i termini "diavolo, diavoli, demonio, demoni", così come l'espressione "Antico Nemico"19, quella che Ignazio ha trattenuto e personalizzato è "nemico del genere umano". Continuiamo allora ad approfondirne il significato, analizzando separatamente il termine "nemico" e la locuzione "natura umana", e poi considerando l'espressione nel suo insieme.

\section{Il nemico nell'orizzonte semantico di Ignazio}

\subsection{Il nemico secondo la tradizione}

L'impiego della parola "nemico" per designare il demonio non è certo un'originalità ignaziana. Nel Nuovo Testamento, Gesù identifica il nemico con il diavolo, quando offre ai suoi discepoli la spiegazione della parabola del grano e della zizzania: "il nemico che ha seminato la zizzania è il diavolo" (Mt 13, 39). La comprensione del demonio come nemico si affermò sempre di più nei primi secoli dell'era cristiana, in particolare quando, affievolendosi le grandi persecuzioni alle quali i cristiani erano stati sottoposti, nacquero le prime forme di vita monastica (anacoretica e cenobitica). Per il fatto stesso di allontanarsi dal mondo e di ritirarsi in luoghi di solitudine, questi uomini e queste donne si esponevano a dure battaglie, in cui il principale nemico era, appunto, il demonio ${ }^{20}$.

Molti autori che, dai primi secoli del cristianesimo fino all'epoca di Ignazio, si sono dedicati alla descrizione della vita nello Spirito, hanno concepito il demonio come "nemico" o "avversario" della vita spirituale, descrivendone le astuzie e proponendo rimedi per disinnescarle. Basti una rapida occhiata ai testi che hanno influenzato direttamente o indirettamente Ignazio per rendersi conto che sono intrisi del linguaggio spirituale bellico, tanto quelli più antichi, come gli scritti di Origene e di Cassiano, quanto quelli più vicini a Ignazio e che lui stesso nomina, come appunto la Vita Christi, la Legenda aurea e il De Imitatione Christi.

Ciò nonostante, solo la considerazione di come questa visione spirituale entri in risonanza con la mentalità cavalleresca medievale, ci permette di

\footnotetext{
${ }^{18}$ La voce "natura" del Diccionario de Autoridades, Tomo IV, (1734), afferma: "Lo mismo que Naturaleza. Es poco usado, y puramente Latino".

${ }^{19}$ In particolare nelle pagine consacrate a san Benedetto e a san Francesco (IACOPO DI VARAZZE, 1995, p. 256-265; 815-829).

${ }^{20}$ Il Dictionnaire de Spiritualité mostra che, secondo la comprensione di quel tempo, il deserto era il luogo in cui il demonio, o i demoni, si rifugiava/no perché era il luogo dove potevano agire indisturbati. La scelta di recarsi nel deserto significava proprio andare a combattere contro i demoni, per portare anche lì la vittoria di Cristo (GUILLAUMONT, 1957, v. 3, p. 190-191).
} 
comprendere fino in fondo la portata che la parola "nemico" assume per Ignazio. Il Medioevo, infatti, è l'epoca che produce e si nutre dei grandi romanzi cavallereschi ${ }^{21}$; è l'epoca in cui la figura del cavaliere si impone come una delle tre figure cardine della società ${ }^{22}$; è l'epoca fondata sul valore dell'onore, di cui il cavaliere è emblema e difensore ${ }^{23}$. Più ancora, nella società cristiana medievale si assiste ad una commistione tra religione e mentalità cavalleresca, al punto che il cavaliere è colui che si occupa della difesa del cristianesimo contro i nemici di esso ${ }^{24}$. Ecco che, quando Erasmo da Rotterdam, alle soglie dell'umanesimo rinascimentale, scriverà l'Enchiridion militis christiani ${ }^{25}$, starà rimodellando "spiritualmente" una figura che era ben presente nella mentalità medievale: quella del soldato di Cristo. L'universo bellico, dunque, apparteneva visceralmente all'orizzonte semantico dell'uomo medievale: la classificazione in amici e nemici era costitutiva del modo in cui l'uomo medievale guardava il mondo e gli uomini, e il cristianesimo ne era fortemente influenzato.

\subsection{Dai nemici di fuori al nemico di dentro}

Il giovane Iñigo era profondamente imbevuto della cultura medievale, tanto più che, essendo figlio dei signori di Loyola, cresciuto alle corti di Arévalo e di Nájera, si forgiava del titolo di hidalgo - cavaliere. Non a caso il Racconto del Pellegrino si apre narrando di una battaglia nella quale il protagonista, pieno di ardore, convince i suoi compagni d'arme a resistere, contro ogni speranza, nella difesa della città di Pamplona (R, n. 1). La ferita alle gambe, provocata da una bombarda, mise in serio pericolo la sua salute fisica, ma non dovette essere meno sconvolgente, per la sua psiche, il constatare che proprio coloro che egli riteneva essere i suoi

\footnotetext{
${ }^{21}$ Per citarne qualcuno, la Chanson de Roland, le varie versioni di Tristano e Isotta, e l'Amadis de Gaula, a cui fa allusione lo stesso Ignazio (R, n. 17).

${ }^{22}$ Jacques Le Goff, nell'introduzione alla raccolta "L'uomo medievale", riporta la tripartizione della società medievale in oratores, bellatores e laboratores formulata dal vescovo Adalberone di Laon nel 1030 (LE GOFF, 1978, p. 12). Per un approfondimento della figura del cavaliere, rinviamo al secondo capitolo di questa raccolta (CARDINI, 1978, p. 81-123).

${ }^{23}$ Michel Zink, nel suo libro L'humiliation, le Moyen Age et nous, mostra come la società medievale fosse fondata sull'imperativo dell'onore, al punto che l'umiliazione - e il potere di infliggerla - era ciò su cui si reggeva il controllo sociale (ZINK, 2017).

${ }^{24} \mathrm{E}$ il tempo delle crociate, della lotta alle eresie, delle guerre confessionali, ecc.

${ }^{25} \mathrm{Ci}$ sono diverse ipotesi sull'influenza che gli scritti di Erasmo avrebbero avuto su Ignazio. (Qess) Egli non ha lasciato nulla di scritto su questo tema, e nemmeno le testimonianze dei primi compagni sono concordi. È probabile che Ignazio abbia letto alcuni testi di Erasmo, per esempio l'Enchiridion militis christiani, già durante la sua permanenza a Barcellona, e che questa lettura abbia profondamente influenzato la sua visione dell'uomo e della relazione tra l'uomo e Dio che emerge nel Principio e Fondamento e nella Contemplazione per raggiungere l'amore. Pedro de Ribadeneira racconta però che lo stesso Ignazio avrebbe preso distanza da questi testi e avrebbe raccomandato ai membri della Compagnia di leggerli con cautela, perché aveva notato che la loro lettura "raffreddava la sua devozione" (Fontes narrativi, IV, Roma: 1965, p. 173.175). Cf. Borras (2007, v. 1, p. 223-224) e Cebollada (1990, p. 49-60).
} 
nemici - i francesi - si presero cura di lui e, "trattandolo con cortesia e amicizia" (R, n. 2), lo riportarono alla sua terra. Possiamo supporre che in quella circostanza cominciò ad incrinarsi la forma mentis che lo induceva a spartire l'umanità in amici e nemici. Questa incrinatura si approfondirà quando, vivendo la prima esperienza di apertura degli occhi ( $R, n .8)$, si renderà conto che il nemico più insidioso non era un nemico umano, ma era colui che minacciava la sua interiorità, introducendo dentro di lui dei pensieri che instillavano l'inimicizia tra lui e Dio, e tra lui e gli uomini. Notiamo però che le categorie cavalleresche, che tanto infiammavano gli animi dell'uomo medievale, tra cui quello di Ignazio, non scompaiono, ma sono riconfigurate dentro un'ottica differente: ciò che muta è il luogo, gli attori e le strategie della battaglia.

Alla luce di questo passaggio interiore di Ignazio possiamo comprendere i due esercizi a impronta militare che egli stesso propone, rispettivamente alla soglia e alla metà della Seconda Settimana: l'Esercizio del Re e la Meditazione delle Due Bandiere. Il primo, intitolato "La chiamata del re terreno aiuta a contemplare la vita del Re eterno", accompagna l'esercitante ad aderire prima al nobile progetto di un re terreno, e poi, attraverso un argomento a fortiori, ad offrirsi radicalmente al servizio del Re eterno. L'orizzonte terreno, per quanto rilevante, viene così dilatato in un orizzonte eterno, e la logica cavalleresca viene assunta e reinterpretata dentro una prospettiva inedita. Questa nuova ottica coinvolge anche la comprensione del nemico. Notiamo infatti che, se l'invito che il re terreno rivolge ai suoi sudditi è quello di andare a conquistare "tutta la terra degli infedeli" (ES, n. 93) - i nemici sono quindi identificati con gli infedeli -, Cristo nostro Signore, Re eterno, invita "tutti gli uomini e ognuno in particolare" a "conquistare tutto il mondo e tutti i nemici" per entrare così nella gloria del Padre (ES, n. 95). Chi sono i nemici se tutti gli uomini sono convocati dal Re eterno per collaborare al suo progetto? È evidente che agli occhi di Cristo nessun uomo è nemico. Forse alcuni uomini si comporteranno nei suoi confronti come nemici, ma non sarà lui a considerarli tali ${ }^{26}$. Ciò detto, Ignazio non escluderà mai che possano verificarsi delle contingenze che richiedano una campagna militare contro gli "infedeli" 27 , ma è diventato consapevole che la battaglia di Cristo si situa ad un altro livello, che le armi da Lui adottate sono altre, e che quando Egli rivolge il suo sguardo agli uomini, vede degli amici che desidera condurre, insieme con sé, al Padre.

Nella Meditazione delle Due Bandiere il linguaggio militare è ancora più esplicito. L'esercitante è invitato a "considerare" i due capitani (Cristo e

\footnotetext{
${ }^{26}$ Questa constatazione ci sembra conforme all'esortazione evangelica: "Amate i vostri nemici..." (Lc 6,27-28).

${ }^{27}$ Emblematica, a questo riguardo, è la lettera in cui Ignazio spiega a Jerónimo Nadal le ragioni per cui vede necessaria una crociata contro i turchi. Cf. Roma, 6 agosto 1552 (Epp. 4, p. 354-359).
} 
Lucifero) che, dopo aver radunato i loro vassalli nei rispettivi campi base (Gerusalemme e Babilonia) e dopo averli istruiti sulle loro strategie opposte, li inviano in battaglia. Gli schieramenti sono quindi ben definiti: in uno c'è Cristo con "tante persone" scelte: "apostoli, discepoli, ecc." (ES, n. 145); nell'altro c'è Lucifero, il "mortale nemico della nostra natura umana" (ES, n. 136), "capo di tutti i nemici" (ES, n. 139), i suoi "demoni" (ES, n. 141). Notiamo però che non c'è un luogo geografico circoscritto in cui i due schieramenti si affrontano: entrambi sono inviati agli uomini e alle donne di ogni tempo e di ogni luogo ${ }^{28}$, i primi per "aiutarli" (ES, n. 146), i secondi per "gettare reti e catene" (ES, n. 142). Il "luogo" deputato a questo combattimento è dunque il cuore dell'uomo ${ }^{29}$. Anche il modo in cui si affrontano questi due schieramenti si rivela particolare: essi infatti non si scontrano direttamente, ma ognuno di essi si rivolge all'interiorità della persona, presentando pensieri che la muovono in una direzione piuttosto che nell'altra. L'esercitante, invitato a considerare questi due schieramenti con le rispettive strategie, si scopre chiamato in causa, perché si rende conto che è proprio il suo cuore ad essere il luogo di combattimento. L'esercizio ha quindi lo scopo di rendere l'esercitante consapevole di questa lotta che si svolge dentro di lui e di affinare la sua sensibilità spirituale affinché impari a smascherare le seduzioni del nemico per non lasciarsi avvinghiare nelle sue reti. Lo potrà fare con il supporto della grazia, che invocherà, e con gli aiuti degli amici di Cristo, che non mancheranno.

\section{La "natura umana"}

\subsection{Un'espressione dibattuta}

Dopo aver mostrato le diverse sfaccettature che assume la parola "nemico" negli scritti di Ignazio, passiamo a considerare l'espressione "natura umana". Essa è stata, fin dai primi secoli dell'era cristiana, sorgente di riflessioni e di scontri per la carica di significati che veicola: basti pensare alla distinzione tra natura umana e natura divina, unite nella persona di Gesù ${ }^{30}$. A seconda poi del modo di concepire la natura umana, derivava un certo modo di comprendere il rapporto tra la natura e la grazia o, detto in altri termini, tra ordine naturale e ordine soprannaturale. È intorno a queste differenti

\footnotetext{
${ }^{28}$ Lucifero sparge i suoi demoni "gli uni in una città, gli altri in un'altra, e così in tutto il mondo, non tralasciando alcuna provincia, luogo, o stato di vita né alcuna persona in particolare" (ES, n. 141). Anche Cristo invia i suoi amici e servitori "in tutto il mondo per diffondere la sua santa dottrina tra le persone di ogni stato e condizione" (ES, n.145).

${ }^{29}$ Inteso come "luogo" dell'intelligenza e degli affetti, quindi della decisione.

${ }^{30}$ Il concetto di unione ipostatica, cioè dell'unione delle due nature - umana e divina nell'unica persona di Cristo, fu affermato al Concilio di Efeso nel 431 e ribadito da quello di Calcedonia nel 451.
} 
concezioni che sono scoppiate delle violente diatribe. Laddove si insisteva maggiormente sulla bontà intrinseca della natura umana e sulla capacità dell'uomo di orientare le proprie forze al bene, si dava meno rilievo alla necessità della grazia. Viceversa, laddove era messa più in evidenza la corruzione della natura umana - attribuita al peccato originale -, il ruolo della grazia risultava preponderante. Il principale sostenitore della prima posizione, portata al suo estremo, fu Pelagio. A lui si oppose Agostino, per mettere in guardia dal rischio dell'autosufficienza. Lo scontro che ne scaturì (siamo nel IV-V secolo), si concluse con la condanna della dottrina pelagiana. La questione rimase comunque sullo sfondo delle riflessioni teologiche successive e, nonostante la posizione equilibrata espressa da Tommaso d'Aquino nel celebre assunto "La grazia non distrugge la natura, ma la perfeziona" (TOMMASO, I, q. 1, a. 8, ad 2), essa si riaccese con inaudita violenza proprio nei tempi in cui visse Ignazio. Fu Lutero, in particolare, a dare voce ad una visione della natura umana "totalmente corrotta" dal peccato originale, motivo per cui invocava la sola gratia. Il Concilio di Trento rispose al fermento riformista affermando la posizione di Tommaso. Le tensioni, però, lungi dall'essere risolte, riaffiorano nel tempo della modernità. Oggi è il concetto stesso di natura ad essere in crisi, ben al di là di una prospettiva teologica: non pochi pensatori contemporanei resistono ad una visione della natura umana considerata come un "dover essere" predeterminato - come un apriori a cui aderire in modo costrittivo -, mentre inneggiano alla libertà dell'uomo considerato in divenire, creatore della propria identità. Questo sguardo tende a contrapporre cultura a natura, storia a natura, libertà a natura ${ }^{31}$.

Non potendo approfondire in questo articolo questi temi vasti e complessi, ci limitiamo ad accennarli, e a chiederci in che misura Ignazio sia stato consapevole delle discussioni del suo tempo e vi abbia preso parte. Benché non si sia mai lanciato nella composizione di opere teologiche - Ignazio è stato fondamentalmente un "pedagogo della vita spirituale", un "mistagogo" -, non si è mai sottratto al confronto con le novità della sua epoca: non a caso scelse di trascorrere lunghi anni di studio a Parigi, che in quei tempi era la principale fucina dell'elaborazione teologica. Non c'è dubbio quindi che Ignazio fosse a conoscenza delle questioni brucianti del suo tempo. Le "Regole per il retto sentire che dobbiamo avere nella Chiesa militante" (ES, n. 352-370) ne sono una prova, in particolare i numeri in cui egli consiglia di "fare molta attenzione al modo di discutere e di parlare" della "predestinazione", della "grazia" e della "fede", per evitare che le persone "si impigriscano e trascurino le opere che conducono alla salvezza e al vantaggio spirituale delle anime" (ES, n. 366-369). Le allusioni alla diatriba luterana sono evidenti. Anche se la preoccupazione di Ignazio

${ }^{31}$ Ebbe una notevole eco, a questo proposito, il dibattito tenutosi nel 1971 a Eindhoven (Olanda) tra Noam Chomsky e Michel Foucault (CHOMSKY; FOUCAULT, 2005). 
non è tanto dottrinale quanto pedagogica - "che le persone semplici non cadano in errore" (ES, n. 367) -, egli assume una posizione chiara sulla questione del rapporto tra natura e grazia: "si può parlare della fede e della grazia (...) ma, particolarmente in questi tempi così pericolosi, non in maniera e in termini tali che le opere e il libero arbitrio ne ricevano danno o non siano tenute in alcun conto" (ES, n. 369). Vediamo già che per Ignazio le opere e il libero arbitrio, cioè la parte che compete all'uomo in ragione della sua natura, giocano un ruolo importante, che non può essere sminuito.

Un'altra pista di approfondimento riguardante la "natura umana", più circoscritta ma non meno stimolante per la nostra ricerca, ci viene dalla constatazione che nel Medioevo questa espressione era usata anche per alludere ai testicoli. Questo collegamento nasce nell'ambito del diritto, di fronte all'esigenza di vietare la pratica della castrazione. Se già nel XIII secolo il re Alfonso X aveva scritto, nelle Siete Partidas, che "colui che castra l'uomo è nemico della natura" ${ }^{32}$ (Setena Partida, Título VIII, lex XIII), in tempi più vicini a Ignazio, Giulio Claro, giurista del ducato di Milano e consigliere del re di Spagna Filippo II, affermava che chi mutila le membra della riproduzione è da punire come "nemico della natura". Esse infatti hanno una "funzione nobile, che è quella di conservare la specie umana" (Sententiae Receptae. Quaest. 68, num. 7). La natura umana sarebbe quindi legata alla procreazione e alla vita, e di conseguenza è nemico della natura umana chiunque ostacoli la procreazione, e più generalmente la vita, schierandosi così dalla parte della morte e della sterilità.

\subsection{La natura umana nella comprensione di Ignazio}

Negli scritti ignaziani i riferimenti più o meno espliciti alla "natura umana" non mancano, e rivelano una concezione fondamentalmente positiva che Ignazio nutriva di essa: negli Esercizi la persona è chiamata a mettere in campo tutte le sue capacità naturali - intelletto, memoria, volontà ${ }^{33}$, immaginazione, sensibilità, ecc. -, per muoverle verso ${ }^{34} \mathrm{l}^{\text {" }}$ oggetto" ricercato, disponendole così a riceverlo ${ }^{35}$. Impiegare al meglio le proprie capacità, secondo Ignazio, non solo non preclude l'azione della grazia, ma al contrario dispone all'accoglienza dell'azione di Dio. Notiamo che, secondo

\footnotetext{
32 "castrans hominem inimicus naturae dicitur".

${ }^{33}$ Intelletto, memoria e volontà sono chiamate da Ignazio le tre "potenze naturali", in linea con la concezione agostiniana (La Trinità, X, 17-18) (ES n. 20, p. 177, 320).

${ }^{34} \mathrm{Il}$ verbo appropriato, utilizzato a più riprese da Ignazio, è "afectar", con un senso attivo (GARCIA DOMINGUEZ, 2007, v. 1, p. 95-102).

35 Tutta la dinamica degli Esercizi Spirituali è un mettere in campo tutte le proprie potenzialità umane, per disporsi a ricevere la grazia: è la dinamica del "preparare e disporre l'anima", del "cercare e trovare la volontà di Dio", annunciata fin dal primo numero degli Esercizi (GIULIANI, 1990, p. 181-191).
} 
Ignazio, le sole "potenze naturali" sono sufficienti a garantire "una sana e buona elezione" (ES, n. 175), a condizione che agiscano "liberamente e tranquillamente" (ES, n. 177), il che si realizza quando sono puramente orientate al "fine per cui l'uomo è nato, cioè per lodare Dio nostro Signore e salvare la propria anima", e "non sono agitate da diversi spiriti" (ES, n. $177)^{36}$. Indipendentemente dal tempo dell'elezione, può capitare che, per un certo periodo, il Signore ritiri la sua grazia sensibile, affidando l'esercitante "alle sue potenze naturali": è il tempo della prova, della desolazione (ES, n. 320). In tale situazione, afferma Ignazio, la persona può comunque "resistere alle varie tentazioni e agitazioni del nemico (...) con l'aiuto di Dio che gli rimane sempre, anche se non lo sente chiaramente" (ES, n. 320). Sembra che in questo caso l'aiuto di Dio, che non manca mai, passi proprio attraverso le potenze naturali dell'uomo.

La visione positiva che Ignazio rivolgeva alla natura umana, e ai mezzi naturali in generale, si evince anche dal fatto che egli non contrappone mai "mezzi naturali" e "mezzi soprannaturali" come se fossero in competizione, come se l'uomo, investendo sui mezzi naturali, recasse un'offesa a Dio inviandogli un segnale di sfiducia. Più ancora, quando Ignazio coglieva che qualcuno tendeva a questa prospettiva dicotomica, non mancava di intervenire, anche vigorosamente. Fu il caso del padre Juan Alvarez che contestava il fatto che la Compagnia avesse fatto ricorso a persone influenti per difendersi da accuse gravi e false, e si chiedeva se un tale gesto non avesse significato "curvare le ginocchia di fronte a Baal". Ignazio, attraverso la penna di Polanco, replico che ragionare in questi termini significava presupporre l'esistenza di due principii separati, "un principio di grazia" e "un principio della natura", e dimenticare che Dio è "autore non solo della grazia ma anche della natura" ${ }^{37}$. La questione della legittimità del servirsi dei mezzi naturali è affrontata anche nelle Costituzioni della Compagnia di Gesù. Nella Parte Decima, dedicata alla conservazione del corpo della Compagnia, si afferma che "i mezzi naturali che dispongono lo strumento di Dio verso il prossimo costituiranno normalmente un aiuto per la conservazione e per lo sviluppo di tutto questo corpo" (Co, n. 814). Quest'affermazione va però compresa dentro la cornice più ampia che la ospita: essa infatti è preceduta dalla dichiarazione che "i mezzi che congiungono lo strumento con Dio e lo dispongono a lasciarsi guidare bene dalla mano divina sono più efficaci di quelli che lo dispongono verso gli uomini" (Co, n. 813), ed è seguita dall'esortazione ad adoperare questi mezzi naturali "unicamente per il servizio di Dio, e non per riporre in essi la propria fiducia" (Co, n. 814). Posto quindi che i doni soprannaturali siano da prediligere rispetto a quelli naturali, anche questi ultimi concorrono al fine della Compagnia, perché Dio nostro Signore "vuole essere glorificato

${ }^{36}$ È quello che Ignazio chiama il "terzo tempo di elezione", o "tempo tranquillo".

${ }^{37}$ Al p. Juan Alvarez, Roma, 18 luglio 1549 (Epp. 2, p. 481-483). 
con quello che Egli dona come Creatore, che è la natura, e con quello che Egli dona come autore della grazia, che è il soprannaturale" (Co, n. 814).

Il fatto che Ignazio volga uno sguardo positivo ai mezzi naturali in generale, e alla natura umana in particolare, non significa che ignori il versante di fragilità e di debolezza che la caratterizza. Questa consapevolezza, però, non provoca in lui risonanze negative, ma al contrario diventa motivo di maggiore attenzione e cura. Talvolta la debolezza della natura umana si manifesta attraverso la diminuzione fisica. In questo caso, le Costituzioni insistono sulla cura delle persone malate e morenti ${ }^{38}$, ma anche sull'importanza di "conservare la natura per il servizio e la lode" di Dio, che gli eccessi di abnegazione potrebbero danneggiare (Co, n. 296). A queste attenzioni fanno eco le numerose lettere in cui Ignazio mostra partecipazione e sollecitudine nei confronti dei compagni provati da infermità o pericoli ${ }^{39}$. Altre volte è la presa in conto della fallibilità insita nella natura umana che richiede di vigilare e di assumere misure conseguenti: è il caso degli avvisi che i novizi devono sentirsi ripetere spesso "affinché, data la condizione della nostra fragile natura umana, non se ne dimentichino" (Co, n. 291), o ancora dell'esortazione rivolta agli studenti di Coimbra ad essere diligenti, per "lottare contro le passioni e le debolezze cui è soggetta la nostra natura" ${ }^{40}$.

Infine, non si può parlare della concezione della natura umana in Ignazio senza citare il Principio e Fondamento. È in questo assioma, posto come una pietra miliare all'inizio del percorso degli Esercizi, che Ignazio offre la sua visione esplicita di chi è l'uomo, e quindi della natura umana: "L'uomo è creato per lodare, fare riverenza e servire Dio nostro Signore" (ES, n. 23). La concezione antropologica veicolata da quest'affermazione è dinamica: l'utilizzo del tempo presente del verbo "creare" - "è creato" lascia intravvedere un movimento di creazione continua, per cui l'uomo è creato da Dio nel presente eterno che sottende il dispiegarsi del tempo; l'originale castigliano "criar", tradotto con "creare", comprende tra i suoi possibili significati anche quello della cura che la madre ha nei confronti del neonato ${ }^{41}$. Questa visione dinamica dell'uomo non riguarda però sol-

\footnotetext{
${ }^{38}$ Cf. Co, n. 89 , p. 272; 595-597.

${ }^{39}$ Tra tutte segnaliamo quella indirizzata al padre Giovanni Battista Viola, Roma, 10 marzo 1554 (Epp. 6, p. 447-450), nella quale Ignazio dispone, con premura, tutte le condizioni per una convalescenza serena.

${ }^{40}$ Agli studenti di Coimbra, Roma, 7 maggio 1547 (Epp. 1, p. 495-510), [2].

${ }^{41}$ Il verbo "criar" - che appare nel libretto degli Esercizi a più riprese (n. 23, 39, 50, ecc.), così come le sue declinazioni nominali "Criador" (n. 5, 15, 16, ecc.) e "criatura" (n. 60, p. 235) - significa in castigliano sia "creare" che "allattare, allevare, nutrire". Oggi coesistono le due forme verbali "crear" e "criar", mentre al tempo di Ignazio esisteva solo la forma "criar", come lo mostra il Tesoro de la Lengua Castellana o Española, in cui la voce "crear" è assente (DE COVARRUBIAS, 1943, p. 369-370). Senza attribuire ad Ignazio intenzioni implicite, sottolineiamo per lo meno questa doppia accezione del verbo, come lo fa Adrien Demoustier (DEMOUSTIER, 2006, p. 167).
} 
tanto la sua origine di essere creato: il Principio e Fondamento sostiene che proprio da questo suo riceversi da un Altro scaturisce il fine dell'uomo, che è appunto "l'essere-per" questo Altro da cui si riceve ${ }^{42}$. Il "lodare, fare riverenza e servire" Dio indica il decentramento di chi non tiene per sé la vita che riceve, ma la dona, alimentando così il flusso vitale che si costituisce nel ricevere e nel donare, o più precisamente nel riceversi da $\mathrm{e}$ nel donarsi a un Altro. In questa visione dinamica, l'uomo è considerato come un essere in divenire, in cammino verso una direzionalità inscritta nella sua stessa natura, che lo spinge ad inventare cammini e forme per conseguire il proprio fine, e così realizzare se stesso - gli Esercizi puntano proprio ad aiutare l'uomo ad orientarsi in verità e a scegliere i mezzi adeguati per arrivarvi.

\section{Il "nemico della natura umana"}

\subsection{Colui che suggerisce una cattiva interpretazione}

Una volta approfondite la concezione di "nemico" e quella di "natura umana" che emergono dagli scritti ignaziani, possiamo intrecciare i campi semantici rispettivi e comprendere meglio cosa intendesse Ignazio quando parlava del "nemico della natura umana", e perché tenesse tanto a questa espressione. Cosa aggiunge di specifico rispetto alla parola "demonio" 43 ? Cosa dice del modo di agire del demonio, di cui è importante essere a conoscenza per poterlo combattere con più incisività?

Una prima considerazione viene dalla visione che Ignazio aveva dell'uomo e che ci ha trasmesso in particolare nel Principio e Fondamento. Se infatti l'uomo è una creatura finalizzata, un essere-per-Dio che procede verso il compimento della sua natura orientando tutte le sue forze a Dio, il nemico della natura umana sarà precisamente colui che farà di tutto per ostacolare questo percorso, per distoglierlo da questa direzionalità. Egli cercherà di far desistere l'uomo dal suo cammino verso Dio, proponendogli distrazioni, insinuando pensieri che rendono insopportabile la fatica e scoraggiandolo per farlo arrestare o addirittura tornare indietro. Le "Regole per sentire e conoscere le varie mozioni che si muovono nell'anima", relative alla Prima e alla Seconda Settimana degli Esercizi (n. 313-336), contengono una dettagliata descrizione delle varie modalità in cui il nemico cerca di compiere questa opera. Se la natura dell'uomo trova il suo fondamento

\footnotetext{
${ }^{42}$ Commentando il Principio e Fondamento, Francisco José Ruiz Pérez concepisce l'uomo come "creatura finalizzata" o parla della sua "finalizzazione creaturale" (RUIZ PEREZ, 2007, v. 2, p. 942-947).

${ }^{43}$ Ricordiamo che Ignazio ha rettificato una lettera scritta da Polanco sostituendo la parola "demonio" con l'espressione "nemico della natura umana".
} 
e la sua realizzazione nella relazione con Dio, il nemico della natura umana procurerà in ogni modo di allentare, se non perfino di recidere questa relazione, insinuando dubbi su Dio e provocando chiusura su di sé, restringimento degli orizzonti alla misura dei propri bisogni, preoccupazione per la propria sopravvivenza. Nei termini di Karl Rahner, il cui pensiero prende le mosse e sviluppa teologicamente le intuizioni ignaziane, l'uomo è un essere trascendentale, cioè costitutivamente aperto al più grande di sé, originariamente capace di percepire l'auto-comunicarsi di Dio (RAHNER, 1998). Il nemico della natura umana cercherà quindi di occultare questo orizzonte trascendente, o per lo meno di presentare agli occhi dell'uomo l'impossibilità di conseguirlo, in modo che quest'ultimo rimanga appiattito sulle sue inquietudini quotidiane. Quando l'uomo sarà diventato un essere ripiegato su di sé, che cerca in se stesso la propria origine e il proprio compimento, sarà allora snaturato, disumanizzato - e il nemico avrà segnato un punto a suo favore ${ }^{44}$.

Altre considerazioni più specifiche ci vengono dai punti in cui Ignazio impiega direttamente l'espressione "nemico della natura umana". Notiamo che essa compare soprattutto laddove la natura umana affiora nel suo lato più fragile. È a questo livello, sembra suggerire Ignazio, che il nemico interviene con più efficacia, perché, sottolineando le debolezze della sua natura e mostrandogliele come ostacoli insormontabili, riuscirà più facilmente a farlo desistere nel cammino verso Dio. Non è un caso che il "nemico della natura umana" sia citato per tre volte di seguito nelle Regole finali della Prima Settimana, in cui, con immagini colorite, si descrive come il nemico faccia leva proprio sulle debolezze dell'uomo: sulla sua paura (ES, n. 325), sulla sua vergogna (ES, n. 326) e sui suoi punti deboli personali (ES, n. 327). Questo modo di procedere del nemico è denunciato da Ignazio anche in una lettera a sor Teresa Rejadell, in cui la invita a contrapporvisi con decisione:

Lei, quindi, di fronte al nemico di tutta la natura umana, che la tenta per toglierle le forze che il Signore le dà e per renderla fiacca e tanto paurosa con insidie e inganni, non osa dire: «Sono desiderosa di servire N. S.», mentre deve dire e proclamare senza timore: «Sono sua serva e morrò piuttosto che rinunciare a servirlo» ${ }^{45}$.

Notiamo quindi che per Ignazio non vi è nessun problema insito nella natura umana, nemmeno nelle debolezze di questa natura, ma che è

\footnotetext{
${ }^{44}$ Edouard Pousset, nella sua lettura della dinamica degli Esercizi ispirata alla Dialectique des Exercices spirituels de saint Ignace de Loyola di Gaston Fessard, interpreta questo cammino come il passaggio graduale da una "posizione di sé da sé e per sé", posizione di peccato, ad una "posizione di sé dall'Altro e per l'Altro", posizione cristica e posizione della pienezza dell'uomo (POUSSET, 1972).

${ }^{45}$ A sor Teresa Rejadell, Venezia, 18 giugno 1536 (Epp. 1, p. 99-107). La citazione si trova alla pagina 103 .
} 
proprio il gioco del nemico quello di far credere che la natura umana sia problematica, inadeguata, portando così l'uomo a considerarla - e a considerarsi di conseguenza - sfavorevolmente, e così a rifiutarla, a rifiutarsi e a rifiutare il Creatore della stessa natura umana. Il nemico insinua quindi un'interpretazione negativa, provocando scoraggiamento e timori che sono nocivi per il cammino dell'uomo ${ }^{46}$. Ignazio non cade in questo inganno - probabilmente vi era caduto una volta per tutte nel tempo degli scrupoli, a Manresa (R, n. 22-25) - e, pur incitando a chiedere l'aiuto del Signore, che non mancherà al momento opportuno, invita a reagire con decisione ${ }^{47}$, scuotendo una volta di più l'uomo che rischia di abbattersi e sollecitandolo a dare credito alle sue stesse potenzialità.

Aggiungiamo che in questa lotta per realizzare, o al contrario ostacolare, la natura umana, l'uomo non è solo con la voce del nemico. C'è anche l'Amico della natura umana, colui che ha manifestato questa amicizia assumendo la natura umana e abitandola in tutti i suoi aspetti, anche quelli più rudi: il Dio incarnato, Gesù Cristo, ci ha mostrato la bellezza e la dignità della natura umana, esibendola nel suo compimento, indicandoci la via per arrivare ad esso e rivelandoci che è possibile giungervi. La via percorsa da lui è stata proprio quella di accogliere la natura umana fino in fondo, senza ribellarsi ad essa, ma facendone uno strumento per il servizio degli uomini e per la gloria del Padre. Egli, vero uomo che "conosce la nostra natura meglio di noi stessi" (ES, n. 89), continua a suggerirci la via, a richiamarci nella buona direzione e ad incoraggiarci nel cammino, con pensieri opposti a quelli del "nemico della natura umana".

\subsection{Il combattimento escatologico nel cuore dell'uomo}

La scelta di Ignazio di adottare l'espressione "nemico della natura umana" può anche essere vista come un modo per uscire da una rappresentazione generalizzata del demonio, ed offrire una rappresentazione più precisa, che aiuti a riconoscerlo nelle sue tattiche e quindi a combatterlo. Cominciamo da quello che questa espressione dice a partire da ciò che non dice: parlando del "nemico della natura umana", Ignazio non parla del demonio come del "nemico di Dio". Solo in una citazione evangelica già riportata,

\footnotetext{
${ }^{46}$ Papa Francesco sottolinea questo aspetto nella lettera apostolica Patris corde: "Il Maligno ci fa guardare con giudizio negativo la nostra fragilità, lo Spirito invece la porta alla luce con tenerezza. È la tenerezza la maniera migliore per toccare ciò che è fragile in noi. Il dito puntato e il giudizio che usiamo nei confronti degli altri molto spesso sono segno dell'incapacità di accogliere dentro di noi la nostra stessa debolezza, la nostra stessa fragilità. Solo la tenerezza ci salverà dall'opera dell'Accusatore (cf. Ap 12,10)" (FRANCESCO, 2020, p. 10). ${ }^{47} \mathrm{~L}$ '"agere contra" raccomandato da Ignazio nella lettera a Teresa Rejadell è un consiglio che esce spesso dalla sua penna, (ES, n. 16, 97, 157, ecc.). Per Ignazio l'uomo, pur confidando in Dio, può e deve reagire con tutte le forze contro le spinte del nemico. È un ulteriore segno della collaborazione all'azione di Dio che l'uomo è chiamato a mettere in atto attraverso le sue capacità naturali.
} 
Ignazio parla del "nemico di Cristo nostro Signore". Lungi dall'affermare che, secondo Ignazio, il demonio sia "amico di Dio", possiamo cogliere in questa opzione una scelta di prendere sul serio il non-dualismo cristiano: il demonio non è "direttamente" nemico di Dio, perché non può nulla contro Dio, perché non è un principio al pari di Dio. È una creatura, e come tale può minacciare l'opera di Dio, ma non Dio stesso. Non c'è dubbio quindi che il demonio si comporti da nemico di Dio, ma appunto non nel senso di un principio che potrebbe ambire a sconfiggere Dio. Non potendo nulla contro Dio, se la prende con la più bella tra tutte le creature - l'uomo appunto.

Potremmo dire che Ignazio prende sul serio il capitolo 12 del libro dell' Apocalisse, in cui è descritta la battaglia definitiva: "il grande drago, il serpente antico, colui che chiamiamo il diavolo e satana e che seduce tutta la terra, fu precipitato sulla terra e con lui furono precipitati tutti i suoi

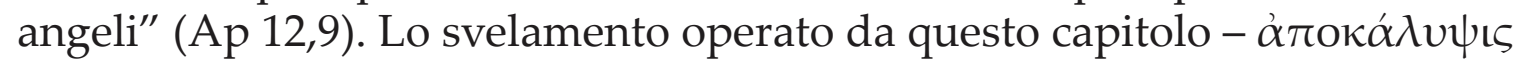
(apokalypsis) significa togliere il velo - spalanca davanti ai nostri occhi il presente eterno di Dio, rivelandoci che nell'eternità la battaglia finale è già stata combattuta, e che l'Agnello con i suoi angeli e i suoi martiri ha già vinto. Il combattimento si gioca ora sulla terra (Ap 12,13.17): la vittoria eterna e definitiva chiede di irradiarsi nel tempo e nello spazio, e questo compimento è rimesso alla libertà e alla responsabilità dell'uomo. Il combattimento escatologico, cioè il combattimento tra la vita e la morte, non è quindi rimandato ad un al di là o ad un a-venire indefiniti: esso si gioca qui ed ora, nel cuore di ogni uomo e di ogni donna ${ }^{48}$, affinché ogni uomo ed ogni donna possano riportare nel loro cuore la vittoria di Cristo, e possano fare del loro cuore - di quel "luogo" che, prima e più di ogni altro luogo sulla terra, è affidato ad ognuno - una porzione del Regno di Dio, piantandovi la bandiera del Risorto.

È vero anche che se il "nemico della natura umana" non può giocare ad armi pari con Dio, non gioca ad armi pari nemmeno con l'uomo, e questa volta in senso contrario, perché l'uomo, da solo, non potrebbe sconfiggere il demonio. Ciò detto, tanto la Meditazione delle Due bandiere quanto più generalmente l'arte del discernimento, di cui è intriso il libretto degli Esercizi, mostrano che il combattimento spirituale non si gioca a due - l'uomo non è solo contro il nemico - ma a tre, perché l'Amico e i suoi alleati vengono in suo aiuto. Il combattimento spirituale non si vince quindi combattendo faccia a faccia contro il nemico - questa è una pericolosa tentazione - ma lasciando entrare dentro di sé il Vincitore. È un combattimento che si vince arrendendosi all'Amico (GIULIANI, 2003, p. 103-120). Non a caso il cammino degli Esercizi è un itinerario in cui la persona fa crescere Cristo in sé, svelando e disinnescando così i tranelli del nemico.

${ }^{48}$ Cf. Meditazione delle Due Bandiere. 
I termini nei quali Ignazio sceglie di parlare del demonio rivelano quindi una chiara prospettiva: senza mai negare le manifestazioni straordinarie in cui il demonio può farsi presente, Ignazio invita a concentrarsi sulla sua azione ordinaria, ben più subdola e pericolosa, perché appunto nascosta e minacciosa per il cuore di ogni persona. Seducendo il cuore, il nemico riesce a deformare lo sguardo sulla realtà e a confondere il bene con il male, spingendo così le persone a compiere azioni malvagie e a diffondere il male nel mondo. Dietro questa concezione di Ignazio, troviamo l'invito a non identificare le persone - o i gruppi di persone - con il male che commettono, o addirittura con il demonio stesso, ma a guardare la realtà nella sua complessità, considerando sempre il combattimento che è in corso nel cuore di ogni uomo e di ogni donna. Identificando il demonio come nemico, Ignazio invita ad uscire da ogni altra indebita identificazione: per il cristiano, al seguito di Cristo, l'unico vero nemico è il demonio: le persone sono "combattute" e a volte rimangono imprigionate dentro le reti del nemico, ma il cristiano è invitato a vederle sempre come amici, e ad aiutarle ad uscire dalle reti nelle quali sono rimaste impigliate.

Ignazio sembra conoscere l'inclinazione dell'uomo ad avere dei nemici, ma questo non sembra stupirlo. Ignazio conosce l'istinto di sopravvivenza dell'uomo, e sa che la tendenza ad avere nemici nasce proprio da questo suo primitivo desiderio di vivere, e dalla sua arcaica paura di perdere la vita. Tutto ciò e tutti coloro che minacciano la vita sono considerati inevitabilmente nemici. Ignazio intuisce che questi impulsi vitali non possono e non devono essere negati o rimossi, perché non può essere rinnegato il desiderio di vivere. Riconfigurando allora il nemico, cioè mostrando che il vero nemico della vita è il demonio, Ignazio offre un nuovo obiettivo verso cui canalizzare la propria aggressività vitale, e propone una nuova concezione dell'uomo e della vita: non sono gli uomini i nemici, e non è nemmeno la vita terrena quel bene ultimo a cui aggrapparsi ad ogni costo. La vita vera è in Dio, ed è possibile sperimentarla già ora, nel cuore del combattimento, ogni volta che la voce del nemico, cioè la voce della morte, è messa a tacere dalla voce della Vita.

\section{Conclusioni}

Abbiamo visto che molte sono le immagini con cui si raffigura il demonio, e molti sono i nomi con cui lo si designa, perché in realtà il demonio non ha un volto e non ha un nome. Egli è contraddizione sussistente: è "angelo", cioè messaggero, che fa di tutto per deteriorare la "buona notizia"; è Lucifero, cioè portatore di luce, la cui principale occupazione consiste nell'estinguere ogni fiammella, affinché le tenebre trionfino. I 
suoi modi di farsi presente variano a seconda delle epoche e dei luoghi, perché si manifesta necessariamente attraverso immagini, pensieri e forme preesistenti nell'orizzonte semantico delle persone su cui spera di fare presa - d'altronde ogni esperienza spirituale è connotata culturalmente e psicologicamente ${ }^{49}$, perché si dà all'interno di certe rappresentazioni, pur eccedendole e trasformandole dal di dentro. Non è dunque un caso che Ignazio abbia scelto il termine "nemico" e più specificamente l'espressione "nemico della natura umana". Questa scelta non è pertinente solo per l'universo medievale, a cui Ignazio apparteneva. Essa è eloquente anche e soprattutto per il mondo moderno, che al tempo di Ignazio esibiva i primi germogli.

Ignazio ha saputo intuire i movimenti dell'epoca che si schiudeva davanti a lui, e si è adoperato a fianco dell'uomo, per favorire l'azione dello Spirito e per ostacolare quella del nemico. Le impalcature sociali e personali che avevano caratterizzato il Medioevo non tenevano più. I vecchi confini si sfaldavano. Nuovi orizzonti si spalancavano di fronte alla scoperta di terre sconosciute, esteriori ma anche interiori. Il mondo assumeva una nuova configurazione e anche l'uomo non si concepiva più come prima: si scopriva sempre più libero e capace di una volontà propria. Si aprivano in quegli anni cammini di soggettivazione, ma non senza derive. Ignazio non demonizza questo processo, anzi lo sposa e lo accompagna. Coglie in esso una potenzialità inaudita, la potenzialità di uomini e di donne pienamente liberi in Dio e davanti a Dio - soggetti realizzati in Lui -, e inventa un itinerario - gli Esercizi Spirituali - affinché l'uomo possa realizzare la sua soggettività. L'uomo, nell'apice della sua intelligenza e della sua affettività liberata, si riconosce creato da un Altro e riconosce che la sua pienezza consiste nel fare della sua vita una lode e un servizio per questo Altro, e liberamente lo sceglie. Attori al cuore di questo processo di soggettivazione ci sono anche Dio e il demonio. Dio è colui che favorirà questo processo, il demonio è colui che lo ostacolerà con tutte le sue forze.

Ecco allora, alla luce degli approfondimenti precedenti, il senso che Ignazio dà all'espressione "nemico della natura umana". Il nemico della natura umana è colui che parla al cuore dell'uomo e della donna per impedire che essi realizzino se stessi, che compiano ciò per cui sono creati, che facciano fiorire la loro natura umana. Facendo leva soprattutto sulla debolezza e sulla vulnerabilità della natura umana, egli suggerirà interpretazioni negative su di essa: insinuerà pensieri di sconforto, di sfiducia, di ripiego sulla propria debolezza; $\mathrm{o}$, al contrario, spingerà a "superarla", a non

\footnotetext{
${ }^{49}$ Questa imprescindibilità dall'ambito culturale del soggetto è sottolineata, in particolare per quanto riguarda i pensieri che vengono da fuori, da José García de Castro (GARCIA DE CASTRO, 2001, p. 84-86).

${ }^{50}$ Evochiamo le spinte contemporanee a superare l'umano (trans-umanesimo).
} 
accoglierla, a non prendersene cura, a sollecitare tutto ciò che sta dalla parte della forza, del potere, della velocità, direzione nella quale sembrano portare alcuni impulsi contemporanei ${ }^{50}$.

Non condanniamo a priori le spinte del mondo attuale, cadendo nella trappola di identificare gruppi o persone con il demonio, ma non dimentichiamo nemmeno che il nemico della natura umana è al lavoro, anche oggi, nel cuore di ciascuno di noi. Siamo in guerra, ci dice Ignazio. Con decisione mettiamoci dalla parte del Vincitore, e con coraggio adoperiamoci perché ad ogni uomo e ad ogni donna siano offerte le condizioni, esteriori ed interiori, per crescere in umanità, per portare a compimento nella propria persona ciò per cui sono creati. Non dimentichiamo di dire a noi stessi e all'umanità di oggi che essere umani è bello, ed è bello proprio con tutte le sue debolezze e vulnerabilità, perché è da lì che nasce la cura, la relazione, il senso ultimo della Vita. Che Dio sia pienamente manifestato - teofania - nella piena manifestazione dell'uomo - antropofania (MORANDI, 1999, p. 380). Che Dio, che in ogni istante vuole e crea la natura umana, sia in essa pienamente manifestato.

\section{Sigle}

$\mathrm{BAC}=$ Obras de san Ignacio de Loyola

Co = Costituzioni della Compagnia di Gesù

Epp $=$ Lettere e Istruzioni

ES = Esercizi Spirituali

$\mathrm{R}=$ Racconto del Pellegrino

\section{Riferimenti}

AGOSTINO. La Trinità. Roma: Città nuova, 1973.

ALFONSO X. Siete Partidas. Madrid: La imprenta Real, 1807.

ARZUBIALDE, S. Casiano e Ignacio. Continuidad y ruptura. In: PLAZAOLA, J. (Ed.). Las fuentes de los Ejercicios Espirituales de San Ignacio: Actas del Simposio Internacional (Loyola, 15-19 septiembre 1997). Bilbao: Mensajero, 1998. p. 123-186.

BORRAS, A. Barcelona. In: GARCIA DE CASTRO J. (Ed.). Diccionario de espiritualidad ignaciana. Bilbao; Santander: Mensajero; Sal Terrae, 2007. v. 1. p. 223-224.

CARDINI, F. Il guerriero e il cavaliere. In: LE GOFF, J. (Ed.). L'uomo medievale. Roma: Laterza, 1978. p. 81-123.

CEBOLLADA, P. Loyola y Erasmo: Aportaciones al estudio de la relación entre ambos. Manresa, Madrid, v. 62, p. 49-60, 1990. 
CHOMSKY, N.; FOUCAULT, M. Della natura umana: Invariante biologico e potere politico. Roma: Derive Approdi, 2005.

CLARUS, J. Sententiae Receptae. Lyon: Horatii Boissat \& Georgii Remeus, 1661.

DE COVARRUBIAS, S. Tesoro de la Lengua Castellana o Española, según la impresión de 1611. Barcelona: S. A. Horta1, 1943. p. 369-370.

DEMOUSTIER, A. Les Exercices Spirituels de Saint Ignace de Loyola: Lecture et pratique d'un texte. Paris: Editions Facultés Jésuites, 2006.

ECHARTE, I. (Ed.). Concordancia ignaciana. Bilbao; Maliaño: Mensajero; Sal Terrae, 1996.

FRANCESCO. Lettera apostolica Patris Corde. Padova: Messaggero, 2020.

GARCIA DE CASTRO, J. El Dios emergente: sobre la "consolación sin causa" [EE, n. 330]. Bilbao; Santander: Mensajero; Sal Terrae, 2001.

GARCIA DOMINGUEZ L. Ma. Afecto. In: GARCIA DE CASTRO, J. (Ed.). Diccionario de espiritualidad ignaciana. Bilbao; Santander: Mensajero; Sal Terrae, 2007. v. 1. p. 95-102.

GARCIA MATEO, R. Imitación de Cristo. In: GARCIA DE CASTRO, J. (Ed.). Diccionario de espiritualidad ignaciana. Bilbao; Santander: Mensajero; Sal Terrae, 2007. v. 2. p. 994-1001.

GIULIANI, M. L'expérience des Exercices spirituels dans la vie. Paris: DDB, 1990.

GIULIANI, M. L'accueil du temps qui vient. Paris: Bayard, 2003.

GUILLAUMONT, A. e C. Démon: littérature monastique. In : VILLER, M. et al. (Ed.). Dictionnaire de Spiritualité. Paris: Beauchesne, 1957. v. 3. p. 189-212.

IACOPO DI VARAZZE. Legenda aurea. Torino: Einaudi, 1995.

IPARRAGUIRRE, I.; DE DALMASES, C.; JURADO, M. R. Obras de san Ignacio de Loyola. Madrid: Biblioteca de Autores Cristianos, 1991.

LE GOFF, J. Introduzione. In: LE GOFF, J. (Ed.). L'uomo medievale. Roma: Laterza, 1978. p. 1-38.

LIES, L. La doctrina de la discreción de espíritus en Ignacio de Loyola y Orígenes de Alejandría. In: PLAZAOLA, J. (Ed.). Las fuentes de los Ejercicios Espirituales de San Ignacio: Actas del Simposio Internacional (Loyola, 15-19 septiembre 1997). Bilbao: Mensajero, 1998. p. 101-121.

LYONNET, ST. Démon: dans l'Ecriture. In : VILLER, M. et al. (Ed.). Dictionnaire de Spiritualité. Paris: Beauchesne, 1957. v. 3. p. 142-152.

MELLONI RIBAS, J. Las influencias cisnerianas de los Ejercicios. In: PLAZAOLA, J. (Ed.). Las fuentes de los Ejercicios Espirituales de San Ignacio: actas del Simposio Internacional (Loyola, 15-19 septiembre 1997). Bilbao: Mensajero, 1998. p. 353- 377.

MORANDI, E. 'Vita Activa': L'azione come antropofania in Hannah Arendt. In: BELARDINELLI, S. Teorie sociologiche dell'azione. Milano: Angeli, 1999. p. 352-419.

PALACIO, C. Mal espíritu. In: GARCIA DE CASTRO, J. (Ed.). Diccionario de espiritualidad ignaciana. Bilbao; Santander: Mensajero; Sal Terrae, 2007. v. 2. p. 1175-1180. 
POUSSET, E. La vie dans la foi et la liberté: essai sur les Exercices Spirituels d'Ignace de Loyola. Paris: C.E.R.P., 1972.

RAHNER, K. Uditori della Parola. Roma: Borla, 1988.

RUIZ PEREZ, F. J. Hombre. In: GARCIA DE CASTRO, J. (Ed.). Diccionario de espiritualidad ignaciana. Bilbao; Santander: Mensajero; Sal Terrae, 2007. v. 2. p. 942-947.

TOMMASO D'AQUINO. Summa Theologiae. Torino : Marietti, 1963.

ZINK, M. L'humiliation, le Moyen Age et nous. Paris: Albin Michel, 2017.

Articolo presentato il 25.03.2021 e approvato il 30.06.2021.

Tiziano Ferraroni SJ è dottore in Teologia al Centro Sèvres di Parigi con una tesi intitolata La brèche intérieure. La vulnérabilité du sujet devant Dieu. Une lecture d'Ignace de Loyola. Attualmente insegna corsi di Teologia Spirituale e di Spiritualità Ignaziana alla sezione san Luigi della Pontificia Facoltà Teologica dell'Italia Meridionale e collabora con il Centro Sèvres di Parigi. Orcid.org/0000-0002-7273-0753. E-mail : ferraroni.t@gesuiti.it

Indirizzo: Comunità san Luigi (gesuiti)

Napoli, via Petrarca 115

80122 - Italia 